\title{
Metrology techniques for the verification of the alignment of the EU gyrotron prototype for ITER
}

\author{
Francisco Sanchez ${ }^{1, *}$, Ferran Albajar ${ }^{1}$, Alessandro Lo Bue ${ }^{1}$, Stephano Alberti ${ }^{2}$, Konstantinos Avramidis ${ }^{4}$, Tullio Bonicelli ${ }^{1}$, \\ Alex Bruschi ${ }^{3}$, Gerd Gantenbein ${ }^{4}$, Jean-Philippe Hogge ${ }^{2}$, Stefan $\mathrm{Illy}^{4}$, Zisis Ioannidis ${ }^{4}$, Alberto Leggieri ${ }^{6}$, François \\ Legrand $^{6}$, Ioannis Pagonakis ${ }^{4}$, Etienne Perial ${ }^{6}$, Tomasz Rzesnicki ${ }^{4}$, Manfred Thumm ${ }^{4}$, and Ioannis Tigelis ${ }^{5}$ \\ ${ }^{1}$ European Joint Undertaking for ITER and the Development of Fusion Energy (F4E), Barcelona, E-08019, Spain \\ ${ }^{2}$ Swiss Plasma Center (SPC), Ecole Polytechnique Fédérale de Lausanne, CH-1015 Lausanne, Switzerland \\ ${ }^{3}$ Institute of Plasma Physics "P.Caldirola", National Research Council of Italy, Milan, Italy \\ ${ }^{4}$ IHM, Karlsruhe Institute of Technology (KIT), Kaiserstr. 12, 76131 Karlsruhe, Germany \\ ${ }^{5}$ Faculty of Physics, National and Kapodistrian University of Athens, Zografou, GR-157 84, Athens, Greece \\ ${ }^{6}$ Thales Electron Devices, 2 rue Marcel Dassault, Vélizy-Villacoublay, F-78141, France
}

\begin{abstract}
The EU gyrotron for the ITER Electron Cyclotron (EC) heating system has been developed in coordinated efforts of the EGYC Consortium, Thales ED (TED) and Fusion for Energy (F4E) and under the supervision of ITER Organization Central Team. After the successful verification of the design of the $1 \mathrm{MW}, 170 \mathrm{GHz}$ hollow cylindrical cavity gyrotron operating at the nominal $\mathrm{TE}_{32,9}$ mode with a short pulse gyrotron prototype at KIT, an industrial $\mathrm{CW}$ gyrotron prototype was manufactured by TED and tested at $\sim 0.8 \mathrm{MW}$ output power and $180 \mathrm{~s}$ pulse duration, which is the limit of the HV power supply currently available at KIT. The experiments are being continued at SPC in 2018 to extend further the pulse duration, taking advantage of the existing $\mathrm{CW}$ full-power capabilities of the gyrotron test facility recently upgraded for the FALCON project. The gyrotron cavity interaction is very sensitive to the alignment of the internal mechanical parts of the gyrotron tube with the magnetic field generated by the superconducting magnet within a typical range of $0.2-0.5 \mathrm{~mm}$. The control of the tolerances and deformations becomes therefore critical to achieving the target performances. With the EU gyrotron prototype it was possible to adjust the alignment of the gyrotron tube with respect to the magnetic field axis during the installation and commissioning phase. The actual shift and tilt movements were verified using advanced metrology methods such as photogrammetry. In this paper, the alignment control techniques and procedures will be discussed also in view of enhancing the reproducibility of gyrotron performance during series production.
\end{abstract}

\section{Introduction}

Europe contributes to the procurement of the highfrequency, high-power gyrotrons for the electron cyclotron resonance heating (ECRH) and current drive (ECCD) system of the ITER tokamak. In this framework, the European GYrotron Consortium (EGYC), in cooperation with Thales Electron Devices (TED) and under the coordination of Fusion for Energy (F4E) is developing the EU $1 \mathrm{MW}-\mathrm{CW}, 170 \mathrm{GHz}\left(\mathrm{TE}_{32,9}\right.$ hollow-cavity) gyrotron prototype.

According to the F4E project planning, the development of the European gyrotron for ITER was organized in two main steps. First of all, a modular short-pulse (SP) prototype was developed by the Karlsruhe Institute of Technology (KIT) in cooperation with TED in order to verify the scientific design of the gyrotron key components with pulses in the ms range. Then, a CW industrial prototype tube (with the same scientific design), TH1509, was developed in order to verify the ITER requirements, in terms of output RF power, efficiency, RF beam quality and pulse length.

The basic scientific design of the $170 \mathrm{GHz}, 1 \mathrm{MW}$ modular SP prototype was successfully verified in multiple experimental campaigns between 2015 and 2017. Based on the SP gyrotron design, the manufacturing of the first industrial $\mathrm{CW}$ prototype gyrotron (TH1509) was completed at TED in 2015. The testing of this $\mathrm{CW}$ industrial prototype was organized in two phases. For the first phase the tube was delivered to KIT in February 2016, where it was operated with the Oxford Instruments (OI) superconducting magnet (see Figure 1 below). Due to the limitation of the currently available HV power supply at KIT, the operation of the gyrotron at KIT is limited at high power to $180 \mathrm{~s}$ pulses. For this reason, after the successful completion of the experiments at $\mathrm{KIT}$, the $\mathrm{CW}$ prototype was delivered to the Swiss Plasma Center (SPC), in Lausanne, where the tube is currently operated with a new cryogen-free superconducting magnet manufactured by Cryogenics (see Figure 2 below). 
One of the key goals at SPC is to extent the pulse length up to one hour. In this work we summarize the importance of the metrology for the correct alignment of the gyrotron with respect to the magnetic field axis (Section 2), we explain the available tools and techniques (Section 3), and we describe the results of the metrology campaigns carried out during the installation and RF high power tests on the TH1509 prototype at KIT and at SPC (Section 4).
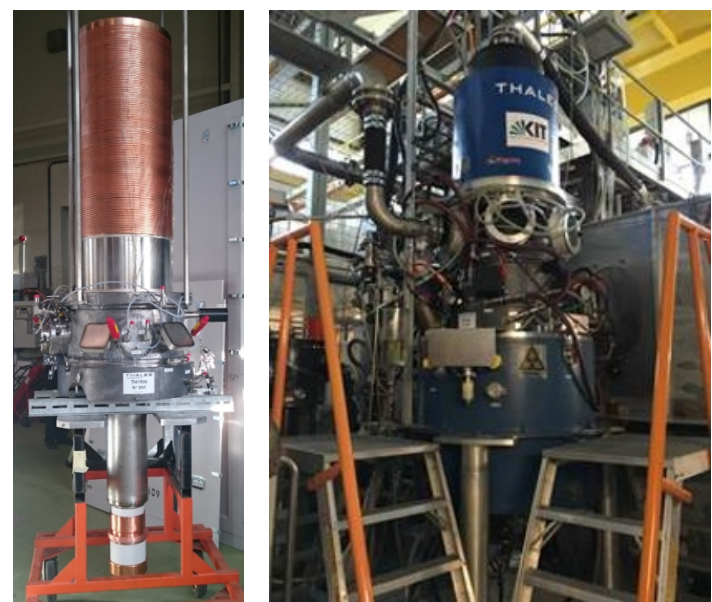

Fig. 1. TH1509 gyrotron prototype (left) and the same gyrotron installed in the KIT test stand (right), in Karlsruhe (Germany).

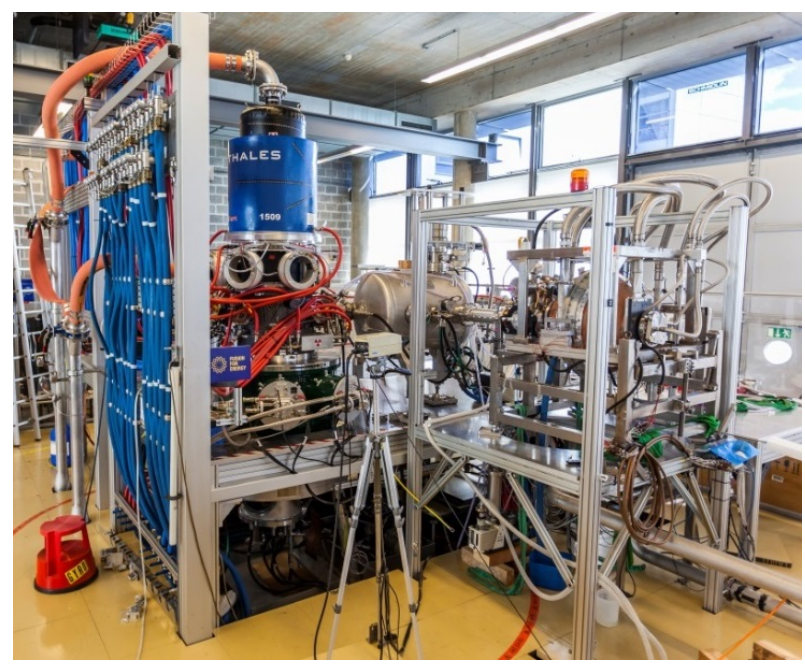

Fig. 2. TH1509 gyrotron prototype installed in the SPC test stand, in Lausanne (Switzerland), with MOU and dummy load.

\section{Importance of alignment sensitivity}

The performance of a gyrotron (see Figure 3, [1]) can be significantly reduced by a misalignment of the electron beam generated in the electron gun and guided by the magnetic field lines of the superconducting (SC) magnet, with respect to the mechanical axis of the gyrotron cavity.

There are numerous sources of misalignment such as the tolerance chain during manufacturing and assembly of the different gyrotron components, stresses release during baking out of the gyrotron, imperfect sitting of the gyrotron on the SC magnet, mechanical vibrations during operation (due to, e.g., water cooling), thermal deformations during RF operation, SC magnet tolerances and deformations, as well as other external effects (e.g., tokamak stray fields and neighbouring SC magnets). As a consequence of these misalignment sources, either the electron beam or the gyrotron cavity can lose concentricity or get tilted with respect to the mechanical references, so that the gyrotron performance may drop. The effect of this misalignment has been theoretically predicted [2] and has recently been measured experimentally [3].

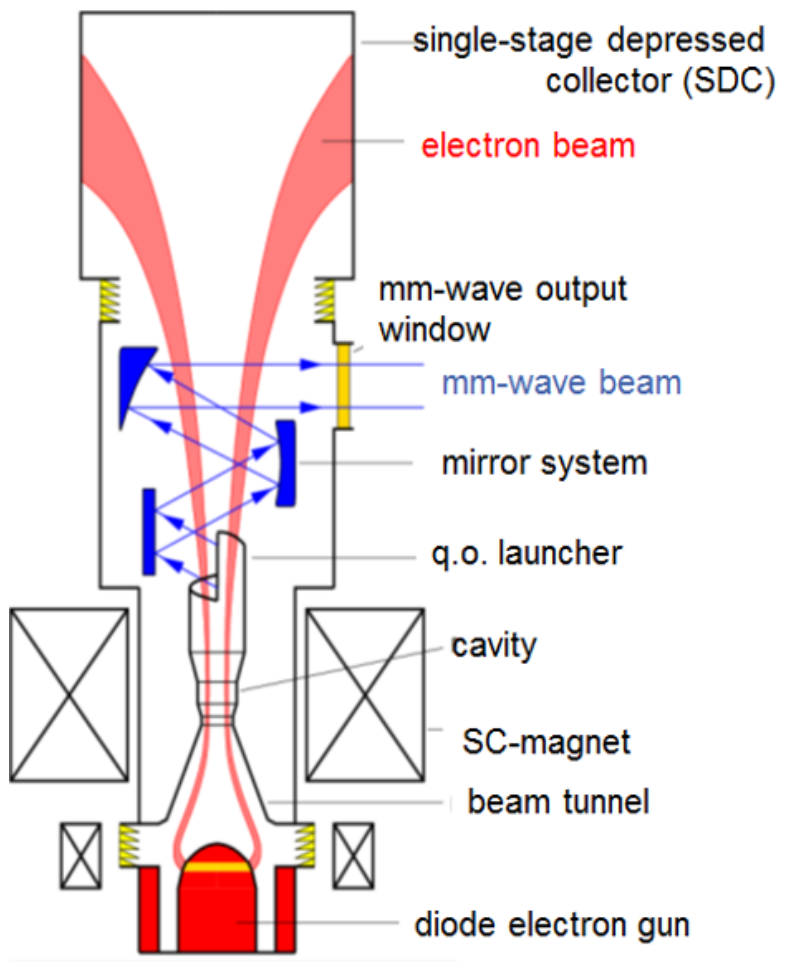

Fig. 3. Gyrotron schematics with electron beam correctly centred with respect to the superconducting (SC) magnet.

RF power measurements were done on the SP gyrotron at KIT when displacing the electron beam by means of additional magnetic fields in two $(\mathrm{x}, \mathrm{y})$ directions orthogonal to the gyrotron vertical axis. The magnetic fields were generated by two dipole coils integrated in the OI SC magnet. The results obtained are summarised on Figures 4 and 5 below: from the optimal position (nominal power extracted from the gyrotron), when the electron beam was deviated by a shift of $\pm 0.25 \mathrm{~mm}$, the output power was reduced by $10 \%$ from the nominal value. Similar results were obtained when measuring the gyrotron efficiency levels. Moreover, the maximum deviation of the tilt angle between the electron beam and the magnetic field angle was also measured experimentally, resulting in maximum acceptable tilt angles of 0.5 degrees (i.e., $\sim 9 \mathrm{mrad}$ ).

By assuming a $10 \%$ maximum of allowable reduction of power and efficiency due to misalignments, the maximum deviation of the electron beam at the cavity would be of $\pm 0.25 \mathrm{~mm}$. The budget of the previously mentioned misalignment sources results in higher overall displacements, therefore measurements and corrections 
are needed. In accordance to the international standards (see BS EN ISO 14253-1, 1999) and F4E procedures, the measurement uncertainty shall be equal or lower than $10 \%$ of the misalignment target, so that for a deviation of $\pm 0.25 \mathrm{~mm}$, the measurement uncertainty shall be less than $25 \mu \mathrm{m}$.

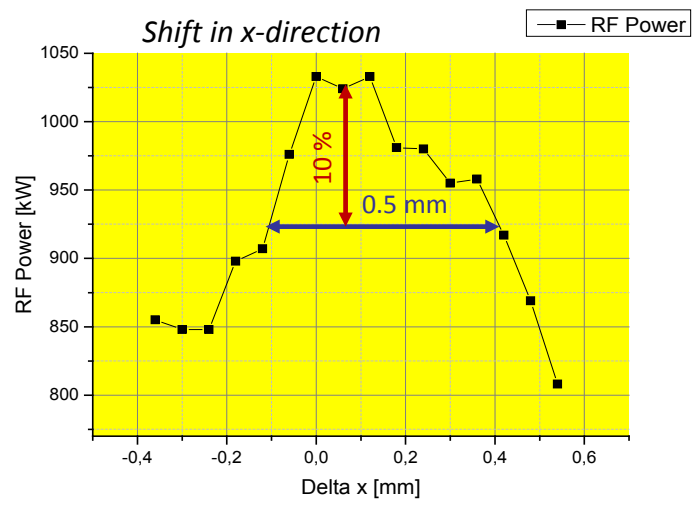

Fig. 4. Influence of the electron beam deviation (x-direction) on the RF power measurements carried out on the SP gyrotron.

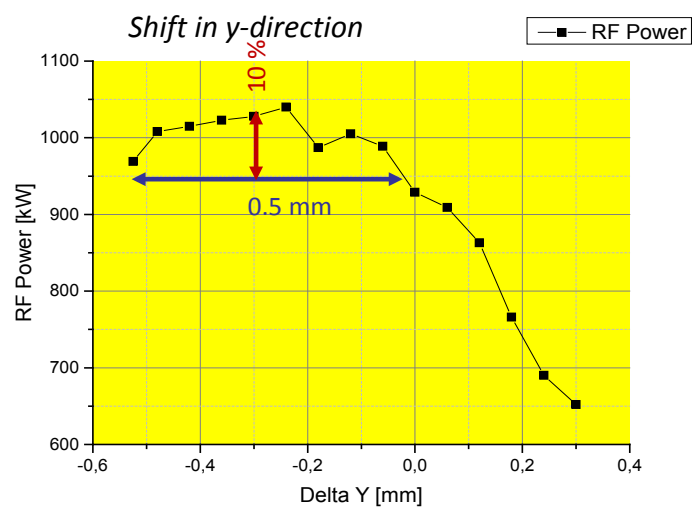

Fig. 5. Influence of the electron beam deviation (y-direction) on the RF power measurements carried out on the SP gyrotron.

Therefore, good alignment between the electron beam and the gyrotron cavity is crucial to obtain a good gyrotron performance, and the use of advanced metrology tools and techniques is highly advisable to comply with the required tolerances and measurement uncertainty. The following sections contain information about the available metrology tools and techniques used on the different surveys done for the EU gyrotron prototype for ITER (1MW-CW, TH1509).

\section{Metrology tools and techniques}

Advanced metrology tools and techniques are recommended to be applied for improving the gyrotron performance, in particular for the ITER gyrotrons, which require to operate in harsh conditions and with tight tolerances. Additionally, it is crucial to control and trace the precise positioning of the gyrotron tube into the magnet in view of ensuring the repeatability of gyrotron performance during the commissioning and maintenance phases. Thus, the following metrology techniques have been applied to the alignment of the EU gyrotron prototype with very good results in terms of repeatability and gyrotron performance.

The first tool used on the EU gyrotron was a laser tracker, whose principle is based on sending a laser ray to a target reference and comparing the forward to the reflected laser ray. This comparison is used to measure variations of the location of objects under analysis and to measure metrology features, such as holes, corners, edges and welding chamfers. The laser tracker is compatible with the required measurement uncertainty $(25 \mu \mathrm{m})$ and offers, as main advantage, the possibility to do measurements of all kinds of metrology features in real time. However, the laser tracker targets are not magnetic proof and they cannot be used when the SC magnet is $\mathrm{ON}$ (so that it is impossible to use the laser tracker when in operation).

A second advanced tool used was photogrammetry, based on triangulation, getting pictures from at least two different locations (named as "lines of sight"), which are mathematically intersected to get the $3 \mathrm{D}$ coordinates of the points of interest. The process outcome are the XYZ coordinates of each one of the points, plus three aiming angles for each picture and camera calibration parameters (including uncertainty estimate). This methodology is even better than the laser tracker in terms of measurement repeatability and compatible with the required uncertainty of $25 \mu \mathrm{m}$. It is ideal to be used in the presence of high magnetic fields, as the target are magnetic and thermal (up to $200{ }^{\circ} \mathrm{C}$ ) proven. The only disadvantage of the photogrammetry is that it cannot be used for measuring metrology features. As conclusion, a combination of a laser tracker metrology campaign with a photogrammetry metrology campaign would be ideal for the case of the ITER gyrotrons. Figure 6 shows the laser tracker and the photogrammetry camera used in the EU gyrotron prototype. Figure 7 shows the sort of images obtained with the photogrammetry.
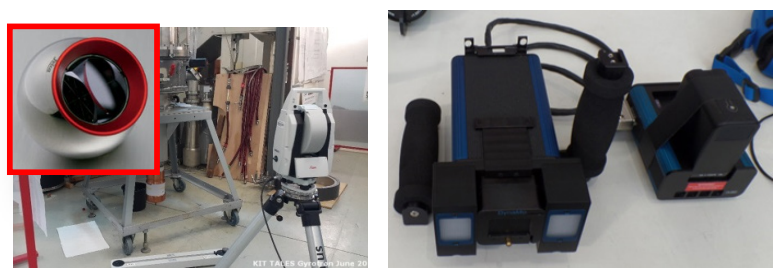

Fig. 6. Laser tracker (and its target), on the left. Camera used for the photogrammetry, on the right.

The metrology surveys shall have a good reference metrology network, based on a reference plane. Ideally, the network and reference plane would have to be established on the most stable component available in the facility. In the case of the EU gyrotron prototype, the SC magnet was chosen to establish a good reference plane and metrology network (see Figure 8). 

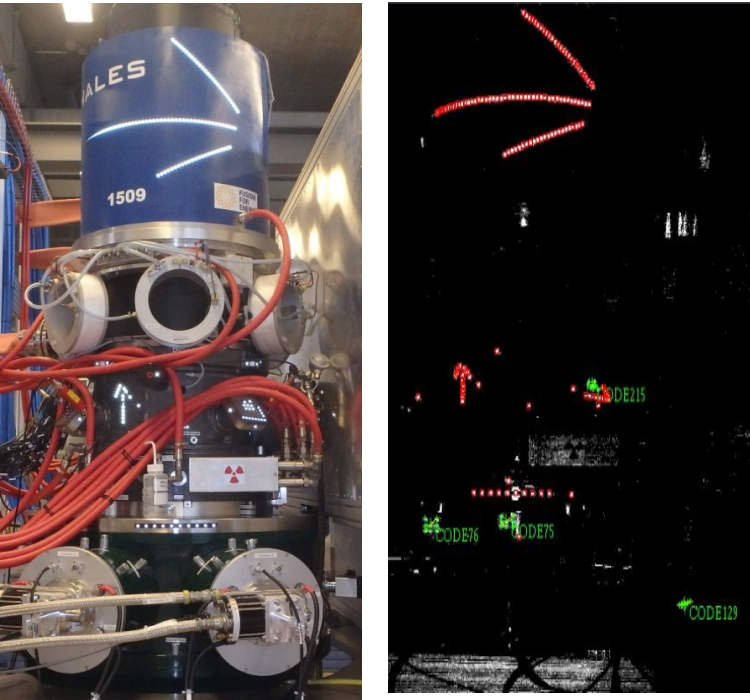

Fig. 7. Normal picture and photogrammetry image obtained in the different targets installed in the EU gyrotron prototype.

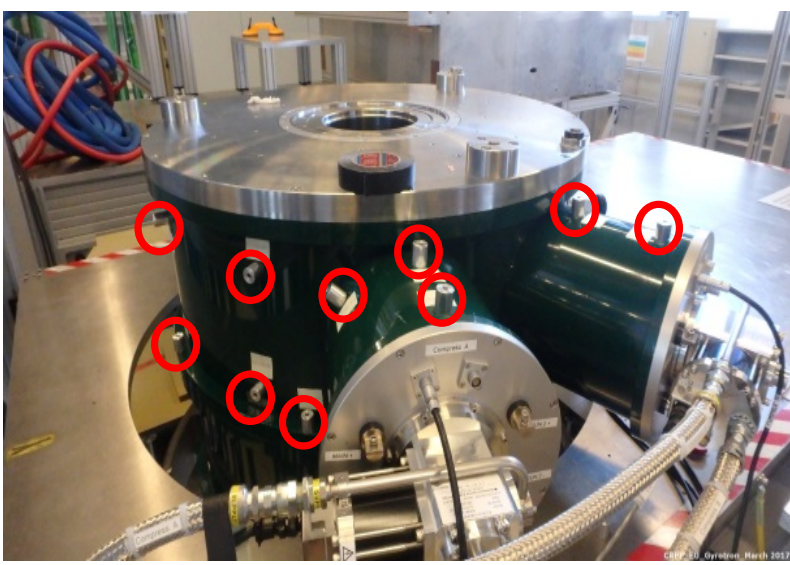

Fig. 8. Metrology network (laser tracker targets depicted in the red circles) as set on the SC magnet at the SPC tests stand (reference place set on the top plate of the magnet).

In order to ensure good reproducibility, it is recommended to plan regular metrology campaigns during the whole life-cycle of the gyrotrons: design, fabrication, assembly, installation, commissioning, operation and maintenance (replacement or refurbishment) for a relevant measurement of the gyrotron performance (in terms of voltage, current, calorimetry measurement of the power and efficiency). Aiming to fulfil the TH1509 operating parameters [4], correction techniques have been applied during design and manufacturing with the inclusion of a centering ring on the gyrotron side and an Adjustable Gyrotron Plate (AGP) on the SC magnet. In addition, mechanical compensations may be carried out to correct manufacturing tolerance chain errors, thermal stresses after baking out, potential misalignments during assembly and installation, influence of water cooling vibrations and thermal deformations after operation, unwanted displacements after possible earthquake events and/or seasonal effects. Finally, the metrology can also verify and ensure proper interface with other optical components (Matching Optical Unit, MOU) and dummy load mirror (used during the acceptance tests) [5]. A new application is under preparation to measure the internal irregularities of the transmission lines (circular corrugated waveguides, $50 \mathrm{~mm}$ diameter). For this application, F4E metrology group has designed a mole probe (see design in Figure 9) consisting of a main body provided with wheels to allow displacements inside the circular waveguides, a cable holder and sensors to measure the possible irregularities. This design is under fabrication and will be used soon for the ITER circular corrugated waveguides.

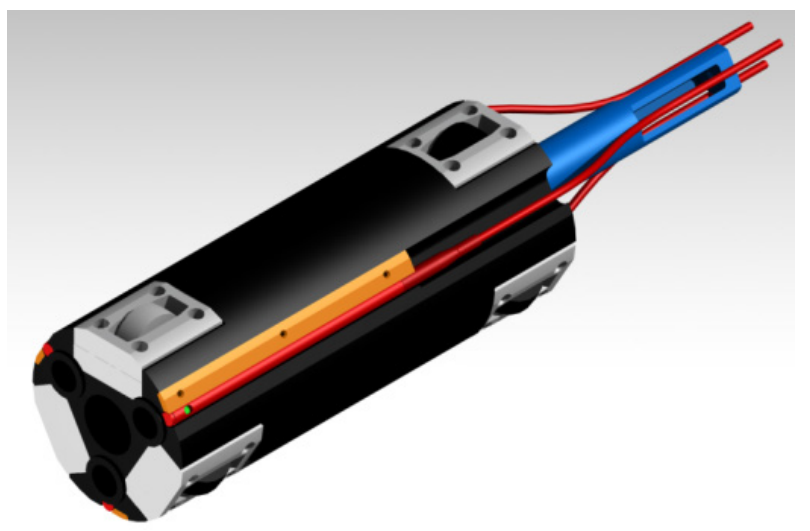

Fig. 9. Mole probe design by F4E to be used to measure irregularities in the ITER circular corrugated waveguides.

\section{Results of the metrology campaigns}

\subsection{Metrology surveys at KIT}

A laser tracker survey combined with photogrammetry was carried out on the TH1509 gyrotron prototype at KIT in June-July 2016. The objective being to measure the concentricity between the anode and the centering ring installed in the gyrotron (interface plane with the SC magnet). The concentricity was successfully verified. In addition, the metrology team measured and verified the alignment between the gyrotron and the SC magnet. In this case, there were some difficulties due to the crowded environment (many components close to the gyrotron) and the high magnetic field, which required to use photogrammetry once the reference plane was established at the bottom of the gyrotron centering ring (see Figure 10). As result of these measurements, a deformation of the centering ring was detected, caused by the weight of the gyrotron when sitting on three bars, which was the only way for the metrology targets to get access to the centring ring. As the survey was nonconclusive, it was recommended to modify the gyrotron sitting jig and to plan for additional metrology surveys. After completing the survey, the gyrotron was tested at KIT and the RF power obtained at the output of the RF window was around $800 \mathrm{~kW}$ for $180 \mathrm{~s}$ [6]. In order to complete the extension of the pulse duration to comply with the ITER requirements [7], the gyrotron prototype was transferred to the SPC test facility in Lausanne [8]. 


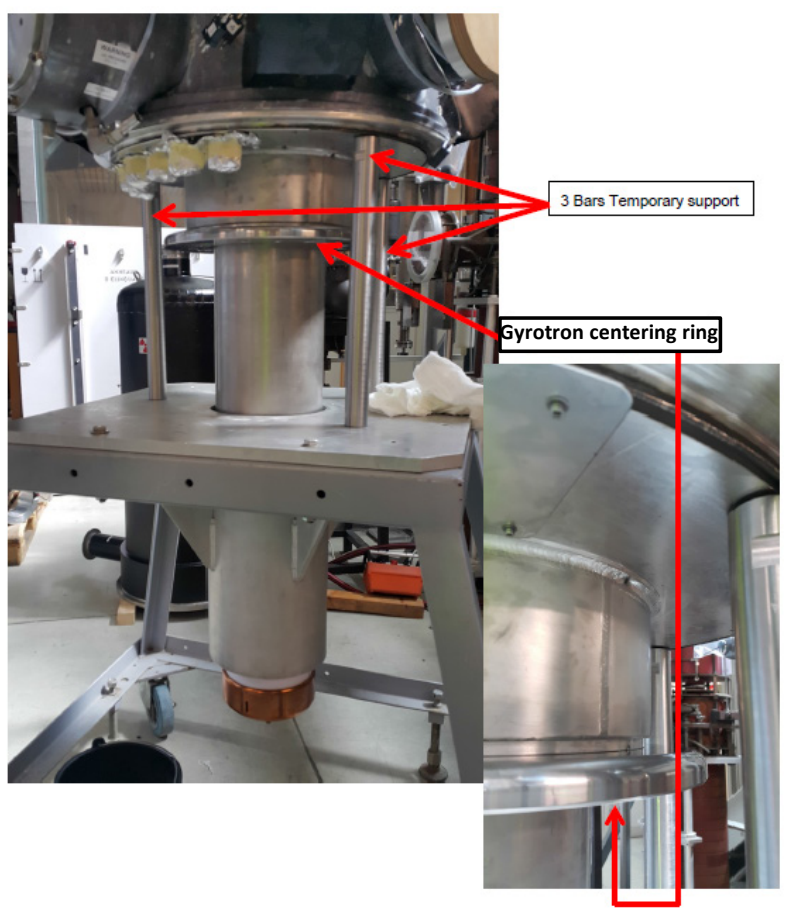

Fig. 10. EU gyrotron prototype (TH1509) at the KIT test stand.

\subsection{Metrology surveys at SPC}

A laser tracker survey also combined with a photogrammetry survey was carried out on the TH1509 gyrotron prototype at SPC in 2017 (in two campaigns: March 2017 for the very short-pulse tests, and October 2017 for the long-pulse tests). The objectives being (similarly to KIT) to verify the concentricity between the anode and the centering ring, and the alignment between the gyrotron electron beam and the SC magnet axis. In this case, the reference plane was set on the top plate (AGP) of the SC magnet and the results showed a deformation of this plate provoked by shims installed during fabrication and installation of the magnet at the SPC facility (see Figure 11).
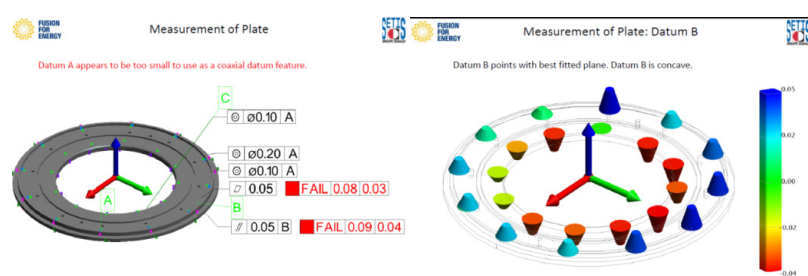

PES

Fig. 11. Reference plane on the SC magnet top plate (AGP) and plate deformations measured by laser tracker.

SPC machined a new plate avoiding shims and ensuring a fat top plate to sit on the gyrotron. The metrology was used both to position correctly the new machined AGP and to align the gyrotron system. The RF tests performed after the metrology survey were successful and very short pulses were produced at the center of the output RF window (see Figure 12).
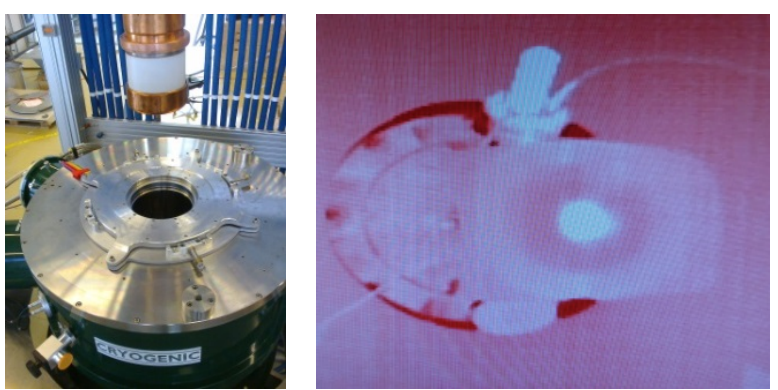

Fig. 12. On the left, SC magnet tope plate (reference plane), together with the XY table while installing the gyrotron. On the right, very short pulse centered at the output RF window.

The second metrology campaign was planned while the gyrotron prototype was in operation for finding the operational maps and RF stability regions which allowed to locate the gyrotron in the center of the region where the RF performance is optimal. This RF operation is usually done with repetitive horizontal displacements of the gyrotron by means of the so-called XY-table [9]. The operation is done with the SC magnet ON (i.e., in the presence of high magnetic fields) and the optimal metrology technique under this conditions was the photogrammetry (magnetic proof targets). Figure 13 shows the photogrammetry targets as seen by a normal picture and with the photogrammetry camera for data processing.

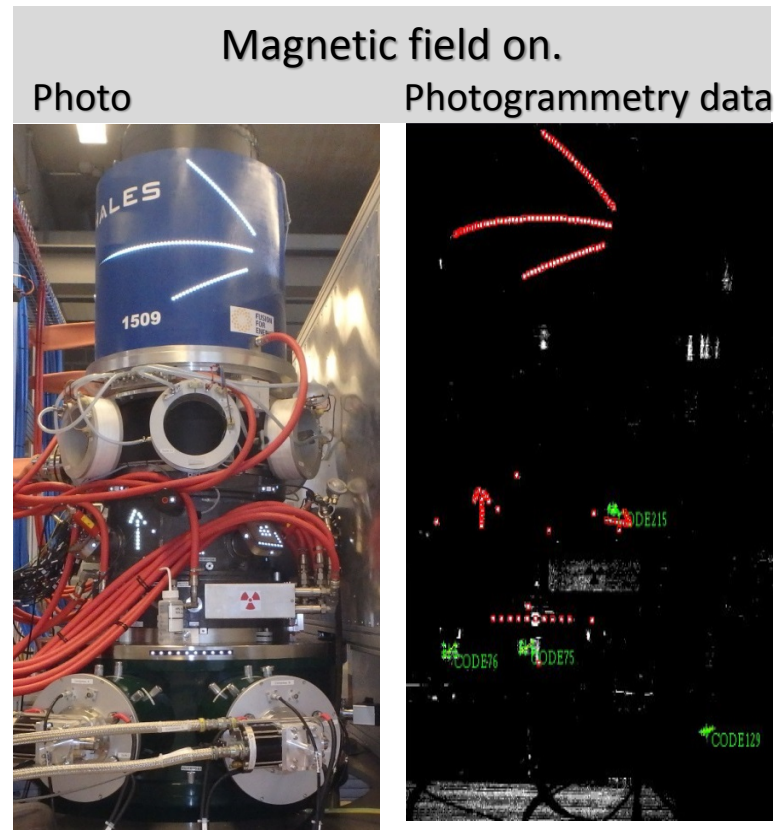

Fig. 13. Left: Photogrammetry targets on the EU gyrotron TH1509. Right: Targets as seen with the photogrammetry camera for data processing.

The metrology campaign results indicated good consistency between the horizontal displacements done on the gyrotron and the measurements taken by the photogrammetry camera. For example, when moving the gyrotron by $1 \mathrm{~mm}$ in $\mathrm{x}$-direction, the metrology targets were measuring a displacement in the same direction in the range 0.936 to $1.015 \mathrm{~mm}$. Additionally, a minor 
rotation of the gyrotron axis was detected with data extracted from the photogrammetry (maybe due to the different degrees of freedom allowed with the XY-table). Figure 14 shows further details on the data measured for this horizontal displacement. The possible tilting of the gyrotron when acting on the XY-table and when operating with the collector water cooling $\mathrm{ON}$ (gyrotron under the effect of vibrations) were also verified.
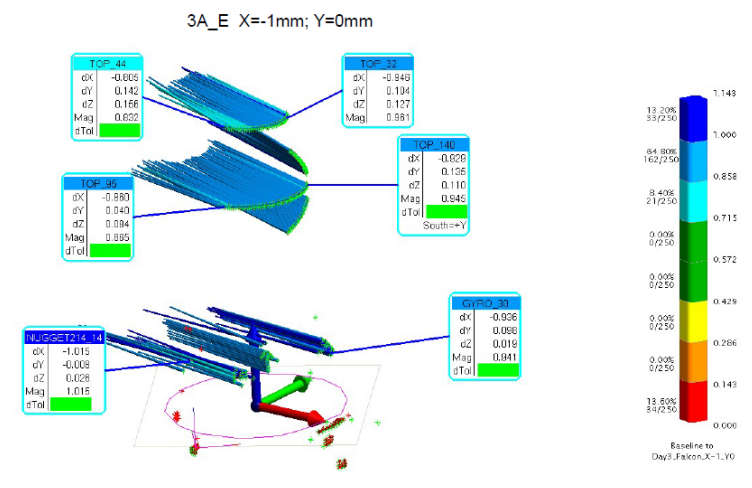

Fig. 14. Photogrammetry data obtained after a horizontal displacement of the gyrotron of $1 \mathrm{~mm}$ in $\mathrm{x}$-direction.

After concluding the metrology campaign and setting the RF stability regions, the gyrotron was moved to the center of the optimal operating region to perform the high-power long-pulse test campaign. The very good behaviour of the gyrotron in terms of vacuum stability and repeatability of the RF performances, allowed to get up to around $800 \mathrm{~kW}$ and pulses of up to 215 seconds. The limitation was found at the maximum energy dissipated in the RF Conditioning Unit (RFCU, aka MOU), whose internal parts are not correctly conditioned for $\mathrm{CW}$ operation. RF power coupled to some internal parts of the RFCU (mirror bellows and flanges) provoked unacceptable temperature increases and the stop of the RF operation when the RF power levels are not optimal yet. The RFCU is currently being modified to cope with $\mathrm{CW}$ operation. A next RF test campaign is planned for September 2018, where the power level and the pulse duration shall be consolidated for the ITER specifications (i.e., reach $1 \mathrm{MW}-\mathrm{CW}$ at 170 $\mathrm{GHz}$, mode $\mathrm{TE}_{32,9}$ and pulse duration of one hour).

\section{Conclusions}

The EU gyrotron prototype for ITER (1 MW-CW, 170 $\mathrm{GHz}$ cylindrical cavity, $\mathrm{TE}_{32,9}$ ) under tests by the EGYC consortium at KIT and SPC has proven operation at power levels around $800 \mathrm{~kW}$ and $215 \mathrm{~s}$ pulse length. In order to get right RF performance, it is important to get a good alignment of the electron beam at the cavity center. In the example shown in the present paper, in order to avoid losing more than $10 \%$ of power/efficiency, the maximum misalignment has to be lower than $\pm 0.25 \mathrm{~mm}$ (i.e., implying a metrology uncertainty of $25 \mu \mathrm{m}$ ). The metrology campaigns done on the TH1509 prototype both with laser tracker and photogrammetry at KIT and at SPC resulted in performing corrections with dipole coils and XY-table. In order to improve the RF performance and reproducibility of gyrotrons, it is recommended to plan for regular metrology campaigns during the different phases of its life-cycle: design, fabrication, assembly, installation, commissioning, operation and maintenance.

\section{Acknowledgement}

This work was done with the shared effort from F4E, the EGYC Consortium and the TED industrial partner.

This publication reflects the views only of the author, and Fusion for Energy cannot be held responsible for any use which may be made of the information contained therein.

\section{References}

1. Courtesy of S. Illy, IHM, Karlsruhe Institute of Technology (KIT).

2. K.A. Avramidis et al., Numerical investigations on the effects of electron beam misalignment on beamwave interaction in a high-power coaxial gyrotron, 38th International Conference on Infrared, Millimeter, and Terahertz Waves (IRMMW-THz), Mainz, Germany, 14-19 September, 2013, Conference Digest.

3. I. Gr. Pagonakis et al., Status of the development of the $170 \mathrm{GHz}, 1 \mathrm{MW}-\mathrm{CW}$ gyrotron, Fusion Engineering and Design, 96-97 (2015), 149-154.

4. Private communication from A. Leggieri (TED) and information from the TH1509 Operation Manual.

5. W. Bin et al, Validation Experiments on the 2-MW CW 170-GHz Load for the European ITER Gyrotron, IEEE Trans. Plasma Sci. 45, Issue 3, 501511 (2017).

6. Z. C. Ioannidis et al., CW experiments with the EU 1-MW, $170-\mathrm{GHz}$ industrial prototype gyrotron for ITER at KIT, IEEE Trans. Electron Devices, vol. 64, no. 9 (2017).

7. C. Darbos et al., Status of the ITER Electron Cyclotron Heating and Current Drive System, Journal of Infrared, Millimeter, and Terahertz Waves, Volume 37 - Number 1 - January 2016, Special Issue: Recent Advances in RF Heating of Plasmas.

8. J-P. Hogge, Status and Experimental Results of the European $1 \mathrm{MW}, 170 \mathrm{GHz}$ Industrial CW Prototype Gyrotron for ITER, presentation at the $41^{\text {st }}$ International Conference on Infrared, Millimeter, and Terahertz Waves (IRMMW-THz), Copenhagen, Denmark, 2016.

9. Z. C. Ioannidis et al., Report of recent experiments with the European $1 \mathrm{MW}, 170 \mathrm{GHz} \mathrm{CW}$ and SP prototype gyrotrons for ITER, EC-20 proceedings, 2018. 